\title{
UV-B induced immunomodulation: a health risk
}

\author{
Johan Garssen, Mary Norval \& Henk Van Loveren
}

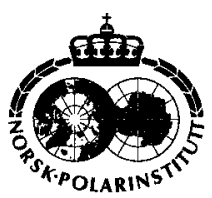

\begin{abstract}
The depletion in stratospheric ozone and changes in life-styles are likely to lead to an increased exposure to sunlight, including the UV-B waveband. Such irradiation may induce immunomodulation and therefore have adverse effects on human health. Alterations in immune responses could affect not only photocarcinogenesis but also resistance to infections, certain allergies and autoimmunity, and vaccination efficacy. In the present study, the risk of increased UV-B exposure has been estimated with respect to the resistance to a bacterial (Listeria monocytogenes) and a viral (herpes simplex virus) infection. The data indicate that suberythemal UV-B irradiation can have significant effects on immune responses to certain infectious diseases in human subjects.
\end{abstract}

J. Garssen \& H. Van Loveren, Laboratory of Pathology and Immunobiology, National Institute of Public Health and Environment, P.O. Box 1. 3720 BA Bilthoven, The Netherlands; M. Norval, Dept. of Medical Microbiology, University of Edinburgh, Edinburgh EH8 9AG, UK.

\section{Introduction}

Ultraviolet radiation (UVR) has been recognized by photobiologists, dermatologists, and oculists as a potential hazard for human health because of its genotoxic, mutagenic, carcinogenic and immunotoxic properties. Its effects include the induction of skin cancers, ocular damage and impairment of resistance to certain infections, with changes in certain allergies and autoimmune diseases also suggested.

Twenty years ago it was demonstrated that UV photons can affect the activity of the immune system through interactions with the skin. This implies that UV not only changes normal cells into cancer cells but also permits the outgrowth of the UV-transformed cells by depressing the immune system (Kripke 1974, 1981). Since then many studies have been performed in order to investigate the immunosuppressive effects of UV-B. Immune function parameters such as contact hypersensitivity, delayed-type hypersensitivity, mixed lymphocyte reactions, mixed skin lymphocyte reactions and antigen presentation, as well as changes in cutaneous phenotypes, have been examined extensively (Hurks, Garssen et al. 1994). In addition, some studies have focused on the consequences of this immunomodulation for resistance to UV induced skin tumours and skin associated infections, such as herpes simplex (Norval et al. 1994; Garssen, Norval et al. 1998; Goettsch, Garssen, Slob et al. 1998; Goettsch, Hurks et al. 1998). Immune response and resistance to herpes simplex virus (HSV) was suppressed by suberythemal doses of UV-B in rodent models and in human subjects. Other skinassociated infections that might be influenced by UV exposure are Leishmania, Mycobacterium leprae and Candida albicans (Giannini 1986; Jeevan \& Kripke 1989; Jeevan et al. 1992; Denkins \& Kripke 1993). Because UV can induce systemic effects in addition to its local effects in the skin, it was suggested that resistance to systemic infections and tumours may also be lowered. Indeed there is now increasing evidence that $\mathrm{UV}-\mathrm{B}$ radiation can alter the immune response to systemic, non-skin associated infections. For example, it was demonstrated that the immune responses and resistance to Mycobacterium bovis were significantly suppressed by exposure (Jeevan \& Kripke 1989; Jeevan et al. 1992). In other studies the immune response to Listeria monocytogenes (a bacterium), Trichinella spiralis (a parasite), HSV (systemic model) and cytomegalo- 
virus (Goettsch, Garssen, De Klerk et al. 1996; Goettsch, Garssen, De Gruijl et al. 1996; Garssen, Van Der Vliet et al. 1995) were affected. In contrast, resistance to Schistosoma (Noonan \& Lewis 1995) and malaria were not altered by suberythemal UV-B exposure.

Based on our data with respect to the $L$. monocytogenes model and studies by De Fabo et al. (1990), an estimation of the risk of exposure of human subjects to increasing UV-B due to a decrease in the ozone layer thickness was calculated (Garssen, Norval et al. 1998; Goettsch, Garssen, Slob 1998; Goettsch, Hurks et al. 1998). The mathematical model included information on the action spectrum for the suppression of contact hypersensitivity (De Fabo \& Noonan 1983) and dose response studies for suppression of $\mathrm{T}$ cell activity in the Listeria infection model, and the estimated biological effective irradiance at certain latitudes (De Fabo et al. 1990).

It is generally agreed that for UV to have a biological effect it must be absorbed initially by a chromophore in the skin that translates the energy into a biochemical signal. This signal will then lead to a change in cellular components, such as cytokines, growth factors, and costimulatory signals, that can modulate the immune response. Various candidates - not mutually exclusive have been proposed to act as the UV photoreceptor. First UV can alter the DNA structure directly (Hurks, Out-Luiting et al. 1995; Vink et al. 1996). Second, UV can be absorbed by cellular components other than DNA, such as lipids in cell membranes, which generate oxidative DNA damage. In addition, lipid peroxidation reactions can trigger intracellular signalling pathways, which in turn may lead to gene activation. A third possible photoreceptor is urocanic acid (UCA), found in the stratum corneum as the trans-isomer. Upon UV irradiation, trans-UCA is converted to the immunosuppressive cis-isomer (De Fabo \& Noonan 1983; Norval 1996). For example, in our studies it became clear that UCA isomerization played a pivotal role in UV-B induced immunosuppression leading to a lowered resistance to Trichinella. Very recently a new mechanism has been implicated in local as well as systemic immunomodulation when it was demonstrated that the sensory nerve system and its neuropeptides, such as calcitonin gene related peptide, are involved in UV induced changes in immunity (Benrath et al. 1995; Niizeki et al. 1997; Garssen, Buckley et al. 1998).
Because many mechanisms might be involved in UV-B induced immunosuppression, for the present paper new risk estimations for decreased ozone layer thickness were performed. In these estimations the action spectrum for DNA damage (Matsunaga et al. 1991), UCA absorption (De Fabo \& Noonan 1983) and mixed skin lymphocyte reactions (Hurks, Out-Luiting et al. 1995) were taken into account instead of the action spectrum for contact hypersensitivity as published earlier. The risk estimations were calculated for systemic infections with L. monocytogenes and HSV.

\section{Methods and results}

Risk assessment comprises four steps: hazard identification, dose-response assessment, exposure assessment, and the ultimate risk characterization. Because experimental data on the effects of UVR on infectious diseases, easily obtainable in laboratory animals, cannot be acquired using human subjects for ethical reasons, and because epidemiological data regarding the incidence of infectious diseases are not readily available, the only way to estimate the risk for humans is to extrapolate from the animal data. The parallelogram approach was used for this, as published earlier (Garssen, Norval et al. 1998; Goettsch, Garssen, Slob et al. 1998; Goettsch, Hurks et al. 1998). In rodent models the effects of UV-B exposure on immune responses to systemic infections with $L$. monocytogenes and HSV were investigated.

Dose response studies in rats infected intravenously with $L$. monocytogenes indicated that $6.8 \mathrm{~kJ} / \mathrm{m}^{2}$ UVB (FS40 lamps) inhibited the specific cellular immune response by $50 \%$. The suppression led to delayed clearance of the bacteria from the spleen. In order to extrapolate from the rat to the human situation, this dose was multiplied by uncertainty factors for inter- and intraspecies differences. In prior studies, using tests for mixed skin lymphocyte reactions and natural killer cell function, it was shown that the average human being was approximately $3-4$ ( 3.85 for mixed skin lymphocyte reaction) times less sensitive than the rat to the effects of UV-B on these parameters. For differences between people, an additional factor of 0.5 was allowed. Thus the extrapolated, i.e. estimated, average dose necessary for the suppression of cellular immunity to L. monocytogenes by 
Table 1. Predicted effect of ozone depletion on solar exposure time necessary for $50 \%$ suppression of cellular immunity to Listeria monocytogenes in human subjects; $40^{\circ} \mathrm{N}$, in July, with a clear sky, around noon. Artificial UV exposure was extrapolated to solar exposure using 3 different action spectra and 1 absorption spectrum. CHS = action spectrum for contact hypersensitivity (De Fabo \& Noonan 1983); DNA = action spectrum for DNA damage (Matsunaga et al. 1991); UCA = Urocanic acid absorption spectrum (De Fabo \& Noonan 1983); MSLR = action spectrum for mixed skin lymphocyte reaction (Hurks, Out-Luiting et al. 1995).

\begin{tabular}{|c|c|c|c|c|}
\hline & \multicolumn{4}{|c|}{$\begin{array}{l}\text { Estimated time in minutes necessary for } 50 \% \\
\text { immunosuppression }\end{array}$} \\
\hline & CHS & DNA & UCA & MSLR \\
\hline No decrease in ozone & 92 & 146 & 119 & 131 \\
\hline $20 \%$ decrease in ozone & 82 & 131 & 106 & 118 \\
\hline
\end{tabular}

$50 \%$ in human subjects was $6.8 \times$ $3.85 \times 0.5=13.1 \mathrm{~kJ} / \mathrm{m}^{2}$ cumulative (Goettsch, Garssen, Slob et al. 1998; Goettsch, Hurks et al. 1998).

One important question is the relevance of the FS40 lamp (broad-band UV-B) to sunlight exposure. In earlier risk assessment studies, the output of the lamp was extrapolated to solar exposure using an action spectrum for suppression of contact hypersensitivity in mice and biological effective irradiance for $50 \%$ immunosuppression in mice at certain latitudes as published by De Fabo \& Noonan (1983) and De Fabo et al. (1990). The exposure times necessary for $50 \%$ suppression of immunity to L. monocytogenes in people at different latitudes were calculated for different amounts of ozone depletion (Garssen, Norval et al. 1998; Goettsch, Garssen, Slob et al. 1998; Goettsch, Hurks et al. 1998). In the present study we performed additional calculations taking other action or absorption spectra into account. These were the absorption spectrum for UCA (De Fabo \& Noonan 1983), the action spectrum for DNA damage (Matsunaga et al. 1991) and the action spectrum for the suppression of the mixed skin lymphocyte reaction (Hurks, Out-Luiting et al. 1995). The results are shown in Table 1.

Recently a model for systemic HSV infection in the rat was developed. The animals were exposed to the FS40 lamps followed by intranasal infection with the virus. HSV is neurotropic and homes to the ganglia and central nervous system. In some animals, depending on the virus load, the infection leads to clinical symptoms that reflect neurological damage (wobbling gait sometimes with paralysis, nervousness, jumpiness). It was found that the virus load increased as the UV-B dose increased, which was also true for the number/

Table 2. Predicted effect of ozone depletion on solar exposure time necessary to increase the incidence of human subjects suffering from clinical symptoms due to herpes simplex virus infection by $10 \% 40^{\circ} \mathrm{N}$, in July, with a clear sky, around noon. Artificial UV exposure was extrapolated to solar exposure using 3 different action spectra and 1 absorption spectrum. CHS = action spectrum for contact hypersensitivity (De Fabo \& Noonan 1983); DNA = action spectrum for DNA damage (Matsunaga et al. 1991); UCA = Urocanic acid absorption spectrum (De Fabo \& Noonan 1983); MSLR = action spectrum for mixed skin lymphocyte reaction (Hurks, Out-Luiting et al. 1995).

Estimated time in minutes for $10 \%$ increase in the number of individuals suffering from clinical symptoms due to systemic herpes simplex virus infection

\begin{tabular}{lcccc}
\cline { 2 - 5 } & CHS & DNA & UCA & MSLR \\
\hline No decrease in ozone & 302 & 478 & 390 & 425 \\
$20 \%$ decrease in ozone & 271 & 429 & 349 & 381 \\
\hline
\end{tabular}


percentage of animals with the clinical symptoms. As in the Listeria model, the data were extrapolated to human subjects using the same uncertainty factors for inter- and intraspecies differences. The extrapolation from artificial UVB exposure (FS40 lamps) to solar exposure was performed using the four different action or absorption spectra as outlined above. The solar exposure required to increase the incidence of clinical symptoms by $10 \%$ (i.e. $10 \%$ more individuals with clinical symptoms) in the general human population was calculated and is shown in Table 2 .

\section{Discussion}

Risk assessment is a process of analysing relevant biological, dose-response, and exposure data for a particular agent in an attempt to establish qualitative and quantitative estimates of adverse effects on human health. Such an assessment of the carcinogenic potential of UV-B radiation is easier to determine than that of UV-B induced immunomodulation and, as a consequence, impaired resistance to certain infections. For risk assessment of the carcinogenic potential of UV-B radiation, the adverse endpoint can be quantified and is beyond dispute (i.e. skin cancer). In contrast, the impairment in T cell immunity to $L$. monocytogenes is difficult to quantify in terms of increased incidence or severity of disease (Garssen, Norval et al. 1998; Goettsch, Garssen, Slob et al. 1998). However with the HSV model the adverse effects can be defined as percentage of individuals with clinical symptoms and/or viral load increments. These endpoints for risk assessment may be more relevant than the impairment of one or two immune functions such as $\mathrm{T}$ cell and natural killer cell activity.

In conclusion, in some animal infection models, UV-B exposure induces decreased immune responses and increased pathogenic load. These results are likely to be relevant for the human situation because many of the immunomodulatory effects described in animals can also be found in people. Determining the exact quantitative relationship between the immunosuppressive effects of UV-B radiation and an increased incidence of infectious diseases in the human population demands a cautious approach; further studies are required. Epidemiological and clinical approaches, as far as these are permitted ethically, may help to clarify the effect of UVR on human infectious diseases and should be areas of future research.

Acknowledgements. - This research was funded by European Community grants EV5V-CT91-0028 and ENV4-CT96-0192 for the EC's Environmental Programme and by the Dutch National Research Programme on Global Air Pollution and Climate Change (NOP: 952276).

\section{References}

Benrath, J., Eschenfelder, C., Zimmermann, M. \& Gillardon, F. 1995: Calcitonin gene-related peptide, substance $P$ and nitric oxide are involved in cutaneous inflammation following ultraviolet irradiation. Eur. J. Pharmacol. 293, 87-96.

De Fabo, E. C. \& Noonan, F. P. 1983: Mechanism of immunosuppression by ultraviolet irradiation in vivo. 1 . Evidence for the existence of a unique photoreceptor in skin and its role in photoimmunology. J. Exp. Med. 158, 84 98.

De Fabo, E. C. Noonan, F. P. \& Frederick, J. E. 1990: Biologically effective doses of sunlight for immune suppression at various latitudes and their relationship to changes in stratospheric ozone. Photochem. Photobiol. 52, 811-817.

Denkins, Y. M. \& Kripke. M. L. 1993: Effect of UV irradiation on lethal infection of mice with Candida albicans. Photochem. Photobiol. 57, 266-271.

Garssen, J., Van Der Vliet, H., De Klerk, A., Goettsch, W., Dormans, J. A. M. A., Bruggeman, C. A., Osterhaus, A. D. M. E. \& Van Loveren, H. 1995: A rat cytomegalovirus infection model as a tool for immunotoxicity testing. Eur. $J$. Pharmacol. Environ. Toxicol. Pharmacol. Sect. 292, 223231.

Garssen. J., Norval. M., El-Ghorr, A., Gibbs, N. K., Jones. C. D., Cerimele, D., De Simone. C., Caffierri, S., DallÁcqua, F., De Gruijl, F. R., Sontag, Y. \& Van Loveren, H. 1998: J. Photochem. Photobiol. B: Biol. 42, 167-179.

Garssen, J., Buckley, T. L. \& Van Loveren. H. 1998: A role for neuropeptides in UVB-induced systemic immunosuppression. Photochem. Photobiol. 68, 205-210.

Giannini, M. S. H. 1986: Suppression of pathogenesis in cutaneous leishmaniasis by UV-irradiation. Infect. Immunol. $51,838-846$.

Goettsch. W., Garssen. J., De Klerk, A., Herremans, M. P. M. T., Dortant, P., De Gruijl, F. R. \& Van Loveren, H. 1996: Effects of ultraviolet-B exposure on the resistance to Listeria monocytogenes in the rat. Photochem. Photobiol. 63, 672679.

Goettsch, W.. Garssen, J.. De Gruijl, F. R. \& Van Loveren, H. 1996: UVB-induced decreased resistance to Trichinella spiralis in the rat is related to impaired cellular immunity. Photochem. Photobiol. 64, 581-585.

Goettsch. W., Garssen, J., Slob, W., De Gruijl, F. R. \& Van Loveren, H. 1998: Risk assessment for the harmful effects of UVB radiation on the immunological resistance to infectious diseases. Env. Health Perspectives 106, 71-77.

Goettsch, W., Hurks, H. M. H., Garssen, J., Mommaas, A. M., Slob, W., Hoekman, J., Pierik, P., Roholl, P. J. M. \& Van Loveren, H. 1998: Comparative immunotoxicology of ultraviolet $B$ exposure. I. Effects of in vitro and in situ 
ultraviolet $B$ exposure on the functional activity and morphology of Langerhans cells in the skin of different species. Br. J. Dermatol. 139, 230-238.

Hurks, M., Garssen, J., Van Loveren, H. \& Vermeer, B.-J. 1994: General aspects of UV-irradiation on the immune system. In G. Jori et al. (eds.): Photobiology in medicine. Pp. 161-175. New York: Plenum Press.

Hurks, H. M. H., Out-Luiting, C., Vermeer, B.-J., Claas, F. H. J. \& Mommaas, A. M. 1995: The action spectra for UV-induced suppression of MLR and MECLR show that immunosuppression is mediated by DNA damage. Photochem. Photobiol. 62 . 449-453.

Jeevan, A. \& Kripke. M. L. 1989: Alteration of immune responses to Mycobacterium bovis BCG in mice exposed chronically to low doses of UV radiation. Cell. Immunol. 145. 2087-2091.

Jeevan, A., Ullich, S. E., Dizon, V. \& Kripke, M. L. 1992: Supernatants of ultraviolet-irradiated keratinocytes decrease the resistance and delayed type hypersensitivity response to Mycobacterium bovis bacillus Calmette-Guernin in mice and impair the phagocytic ability of macrophages. Photodermatol. Photoinmunol. Photomed. 9, 255-263.
Kripke. M. L. 1974: Antigenicity of murine tumors induced by ultraviolet light. J. Natl. Cancer Inst. 53, 1333-1336.

Kripke. M. L. 1981: Immunologic mechanisms in UV-radiation carcinogenesis. Adv. Cancer Res. 34, 69-106.

Matsunaga, T., Hieda, K. \& Nikaido, O. 1991: Wavelength dependent formation of thymine dimers and $(6-4)$ photoproducts in DNA by monochromatic ultraviolet light ranging from 150 to $365 \mathrm{~nm}$. Photochem. Photobiol. 54, 403-410.

Niizeki, H., Alard, P. \& Streilein, J. W. 1997: Calcitonin Generelated Peptide is necessary for ultraviolet B-impaired induction of contact hypersensitivity. J. Immunol. 159, 5183-5186.

Noonan, F. P. \& Lewis, F. A. 1995: UVB-induced immune suppression and infection with Schistosoma mansoni. Photochem. Photobiol. 61, 99-105.

Norval, M. 1996: Chromophore for UV-induced immunosuppression : urocanic acid. Photochem. Photobiol. 63, 386-390.

Norval, M., El-Ghorr, A., Garssen, J., \& Van Loveren, H. 1994: The effects of ultraviolet light irradiation on viral infections. Br. J. Dermatol. 130, 693-700.

Vink, A. A., Yarosh, D. B. \& Kripke, M. L. 1996: Chromophore for UV-induced immunosuppression: DNA. Photochem. Photobiol. 63, 383-386. 